\title{
Canonical Data Structure for Interval Probe Graphs
}

\author{
Ryuhei Uehara*
}

$2004 / 12 / 24$

\begin{abstract}
The class of interval probe graphs is introduced to deal with the physical mapping and sequencing of DNA as a generalization of interval graphs. The polynomial time recognition algorithms for the graph class are known. However, the complexity of the graph isomorphism problem for the class is still unknown. In this paper, extended $\mathcal{M P} \mathcal{Q}$-trees are proposed to represent the interval probe graphs. An extended $\mathcal{M} \mathcal{P} \mathcal{Q}-$ tree is canonical and represents all possible permutations of the intervals. The extended $\mathcal{M P \mathcal { Q }}$-tree can be constructed from a given interval probe graph in $O\left(n^{2}+m\right)$ time. Thus we can solve the graph isomorphism problem for the interval probe graphs in $O\left(n^{2}+m\right)$ time. Using the tree, we can determine that any two nonprobes are independent, overlapping, or their relation cannot be determined without an experiment. Therefore, we can heuristically find the best nonprobe that would be probed in the next experiment. Also, we can enumerate all possible affirmative interval graphs for any interval probe graph.
\end{abstract}

Keywords: Bioinformatics, graph isomorphism, interval probe graph.

\section{Introduction}

The class of interval graphs was introduced in the 1950's by Hajös and Benzer independently. Since then a number of interesting applications for interval graphs have been found including to model the topological structure of the DNA molecule, scheduling, and others (see $[8,17,5]$ for further details). The interval graph model requires all overlap information. However, in many cases, only partial overlap data exist. The class of interval probe graphs is introduced by Zhang in the assembly of contigs in physical mapping of DNA, which is a problem arising in the sequencing of DNA (see $[21,23,22,17]$ for background). An interval probe graph is obtained from an interval graph by designating a subset $P$ of vertices as probes, and removing the edges between pairs of vertices in the remaining set $N$ of nonprobes. That is, on the model, only partial overlap information (between a probe and the others) is given. From the graph theoretical point of view, interval probe graphs are related to tolerance graphs [10, Section 4], and recently, the notion is extended to the chordal probe graphs $[9,20]$. On the other hand, from the practical point of view, a few efficient algorithms for the class are known; the recognition algorithms $[13,16,12]$, and an algorithm for finding a tree 7 -spanner (see [4] for details). The recognition algorithm in [13] also gives a data structure that represents all possible permutations of the intervals of an interval probe graph.

A data structure called $\mathcal{P} \mathcal{Q}$-trees was developed by Booth and Lueker to represent all possible permutations of the intervals of an interval graph [3]. Korte and Möhring simplified their algorithm by introducing $\mathcal{M P} \mathcal{Q}$ trees [14]. An $\mathcal{M P} \mathcal{Q}$-tree is canonical; that is, given two interval graphs are isomorphic if and only if their corresponding $\mathcal{M P Q}$-trees are isomorphic. However, there are no canonical $\mathcal{M P Q}$-trees for interval probe graphs. In general, given an interval probe graph, there are several affirmative interval graphs those are not isomorphic, and their interval representations are consistent to the interval probe graph.

In this paper, we extend $\mathcal{M P} \mathcal{Q}$-trees to represent interval probe graphs. The extended $\mathcal{M P} \mathcal{Q}$-tree is canonical for any interval probe graph, and the tree can be constructed in $O\left(n^{2}+n m\right)$ time. Thus the graph isomorphism problem for interval probe graphs can be solved in $O\left(n^{2}+n m\right)$ time. From the theoretical point of view, the complexity of the graph isomorphism of interval probe graphs was not known (see [19] for related results and references). Thus the result improves the upper bound of the graph classes such that the graph isomorphism problem can be solved in polynomial time.

${ }^{*}$ School of Information Science, JAIST, Ishikawa, Japan. uehara@jaist.ac.jp. 
From the practical point of view, the extended $\mathcal{M P \mathcal { Q }}$-tree is very informative, which is beneficial in the Computational Biology community. The extended $\mathcal{M P} \mathcal{Q}$-tree gives us the information between nonprobes; the relation of two nonprobes is either (1) independent (they cannot overlap with each other), (2) overlapping, or (3) not determined without experiments. Hence, to clarify the structure of the DNA sequence, we only have to experiment on the nonprobes in the case (3). Moreover, given extended $\mathcal{M P} \mathcal{Q}$-tree, we can find the nonprobe $v$ that has most nonprobes $u$ such that $v$ and $u$ are in the case (3) in linear time. Therefore, we can heuristically find the "best" nonprobe to fix the structure of the DNA sequence.

\section{Preliminaries}

The neighborhood of a vertex $v$ in a graph $G=(V, E)$ is the set $N_{G}(v)=\{u \in V \mid\{u, v\} \in E\}$, and the degree of a vertex $v$ is $\left|N_{G}(v)\right|$ and denoted by $\operatorname{deg}_{G}(v)$. For the vertex set $U$ of $V$, we denote by $N_{G}(U)$ the set $\{v \in V \mid v \in N(u)$ for some $u \in U\}$. If no confusion can arise we will omit the index $G$. Given graph $G=(V, E)$, its cograph is defined by $\bar{E}=\{\{u, v\} \mid u, v \in V$ and $\{u, v\} \notin E\}$, and denoted by $\bar{G}=(V, \bar{E})$. A vertex set $I$ is independent set if $G[I]$ contains no edges, and then the graph $\bar{G}[I]$ is said to be a clique.

For a given graph $G=(V, E)$, a sequence of the vertices $v_{0}, v_{1}, \cdots, v_{l}$ is a path, denoted by $\left(v_{0}, v_{1}, \cdots, v_{l}\right)$, if $\left\{v_{j}, v_{j+1}\right\} \in E$ for each $0 \leq j \leq l-1$. The length of a path is the number of edges on the path. For two vertices $u$ and $v$, the distance of the vertices is the minimum length of the paths joining $u$ and $v$. A cycle is a path beginning and ending with the same vertex. An edge which joins two vertices of a cycle but is not itself an edge of the cycle is a chord of that cycle. A graph is chordal if each cycle of length at least 4 has a chord. Given graph $G=(V, E)$, a vertex $v \in V$ is simplicial in $G$ if $G[N(v)]$ is a clique in $G$. The following lemma is a folklore:

Lemma 1 Given chordal graph, all simplicial vertices can be found in linear time.

Proof. It is well known that a graph $G=(V, E)$ is chordal if and only if it is an intersection graph of subtrees of a tree $T(G)$. It can be assumed that the vertices of $T(G)$ are the maximal cliques of $G$, and the subtrees $T_{v}$ for $v \in V$ are defined by the occurrences of $v$ in the maximal cliques of $G$. Such a tree $T(G)$ is called a clique tree of $G$, and can be found in linear time $[11,2,7]$ (see $[5$, p. 7] for further details). In the clique tree $T(G)$, each vertex $v$ is simplicial if and only if $v$ appears exactly once in $T(G)$. Thus we can find all simplicial vertex in linear time.

The ordering $\left(v_{1}, \cdots, v_{n}\right)$ of the vertices of $V$ is a perfect elimination ordering of $G$ if the vertex $v_{i}$ is simplicial in $G\left[\left\{v_{i}, v_{i+1}, \cdots, v_{n}\right\}\right]$ for all $i=1, \cdots, n$. Then a graph is chordal if and only if it has a perfect elimination ordering (see, e.g., [5, Section 1.2] for further details).

Two graphs $G=(V, E)$ and $G^{\prime}=\left(V^{\prime}, E^{\prime}\right)$ are isomorphic if and only if there is a one-to-one mapping $\phi: V \rightarrow V^{\prime}$ which satisfies $\{u, v\} \in E$ if and only if $\{\phi(u), \phi(v)\} \in E^{\prime}$ for every pair of vertices $u$ and $v$. We denote by $G \sim G^{\prime}$ if $G$ is isomorphic to $G^{\prime}$. The mapping $\phi$ is called an isomorphism from $G$ to $G^{\prime}$. Given graphs $G$ and $G^{\prime}$, graph isomorphism problem is the problem to determine if $G \sim G^{\prime}$.

\subsection{Interval graph representation}

A graph $(V, E)$ with $V=\left\{v_{1}, v_{2}, \cdots, v_{n}\right\}$ is an interval graph if there is a set of intervals $\mathcal{I}=\left\{I_{v_{1}}, I_{v_{2}}, \cdots, I_{v_{n}}\right\}$ such that $\left\{v_{i}, v_{j}\right\} \in E$ if and only if $I_{v_{i}} \cap I_{v_{j}} \neq \emptyset$ for each $i$ and $j$ with $1 \leq i, j \leq n$. We call the set $\mathcal{I}$ of intervals interval representation of the graph. For each interval $I$, we denote by $R(I)$ and $L(I)$ the right and left endpoints of the interval, respectively (hence we have $L(I) \leq R(I)$ and $I=[L(I), R(I)]$ ).

A graph $G=(V, E)$ is an interval probe graph if $V$ can be partitioned into subsets $P$ and $N$ (corresponding to the probes and nonprobes) and each $v \in V$ can be assigned to an interval $I_{v}$ such that $\{u, v\} \in E$ if and only if both $I_{u} \cap I_{v} \neq \emptyset$ and at least one of $u$ and $v$ is in $P$. In this paper, we assume that $P$ and $N$ are given, and then we denote by $G=(P, N, E)$. By definition, $N$ is an independent set, $G[P]$ is an interval graph, and $G[P \cup\{v\}]$ is also an interval graph for any $v \in N$. Let $G=(P, N, E)$ be an interval probe graph. Let $E^{+}$be a set of edges $\left\{t_{1}, t_{2}\right\}$ with $t_{1}, t_{2} \in N$ such that there are two probes $v_{1}$ and $v_{2}$ in $P$ such that $\left\{v_{1}, t_{1}\right\} \in E$, $\left\{v_{1}, t_{2}\right\} \in E,\left\{v_{2}, t_{1}\right\} \in E,\left\{v_{2}, t_{2}\right\} \in E$, and $\left\{v_{1}, v_{2}\right\} \notin E$. Intuitively, nonprobes $t_{1}$ and $t_{2}$ are joined by an edge in $E^{+}$if (1) there are two independent probes $v_{1}$ and $v_{2}$, and (2) both of $v_{1}$ and $v_{2}$ overlap $t_{1}$ and $t_{2}$. In the case, we can know that intervals $t_{1}$ and $t_{2}$ have to overlap without experiment. Each edge in $E^{+}$is called an enhanced edge, and the graph $G^{+}:=\left(P, N, E \cup E^{+}\right)$is said to be an enhanced interval probe graph. It is known 
that an interval probe graph is weakly chordal [18], and an enhanced interval probe graph is chordal [21, 23]. For further details and references can be found in [5, 17].

For a given interval probe graph $G$, an interval graph $G^{\prime}$ is said to be affirmative if and only if $G^{\prime}$ gives one possible interval representations for $G$. More precisely, a given interval probe graph $G=(P, N, E)$, an interval graph $G^{\prime}=\left(V, E^{\prime}\right)$ is affirmative if and only if there is a partition of $V$ into $P$ and $N$ such that $E=\left\{\{u, v\} \mid\{u, v\} \in E^{\prime}\right.$ and at least one of $u$ and $v$ is in $\left.P\right\}$. In general, for an interval probe graph $G$, there are several non-isomorphic affirmative interval graphs. For a given interval probe graph $G=(P, N, E)$, the affirmative interval graph $G^{\prime}$ is also said to be affirmative to the corresponding enhanced interval probe graph $G^{+}=\left(P, N, E \cup E^{+}\right)$.

\section{$2.2 \quad \mathcal{P} \mathcal{Q}$-trees and $\mathcal{M P Q}$-trees}

$\mathcal{P} \mathcal{Q}$-trees were introduced by Booth and Lueker [3], and which can be used to recognize interval graphs as follows. A $\mathcal{P} \mathcal{Q}$-tree is a rooted tree $T$ with two types of internal nodes: $\mathcal{P}$ and $\mathcal{Q}$, which will be represented by circles and rectangles, respectively. The leaves of $T$ are labeled 1-1 with the maximal cliques of the interval graph $G$. The frontier of a $\mathcal{P} \mathcal{Q}$-tree $T$ is the permutation of the maximal cliques obtained by the ordering of the leaves of $T$ from left to right. $\mathcal{P} \mathcal{Q}$-tree $T$ and $T^{\prime}$ are equivalent, if one can be obtained from the other by applying the following rules a finite number of times; (1) arbitrarily permute the successor nodes of a $\mathcal{P}$-node, or (2) reverse the order of the successor nodes of a $\mathcal{Q}$-node. In [3], Booth and Lueker showed that a graph $G$ is an interval graph if and only if there is a $\mathcal{P} \mathcal{Q}$-tree $T$ whose frontier represents a consecutive arrangement of the maximal cliques of $G$. They also developed an $O(|V|+|E|)$ algorithm that either constructs a $\mathcal{P} \mathcal{Q}$-tree for $G$, or states that $G$ is not an interval graph. If $G$ is an interval graph, then all consecutive arrangements of the maximal cliques of $G$ are obtained by taking equivalent $\mathcal{P} \mathcal{Q}$-trees.

Lueker and Booth [15], and Colbourn and Booth [6] developed labeled $\mathcal{P} \mathcal{Q}$-trees in which each node contains information of vertices as labels. Their labeled $\mathcal{P} \mathcal{Q}$-trees are canonical; given interval graphs $G_{1}$ and $G_{2}$ are isomorphic if and only if corresponding labeled $\mathcal{P} \mathcal{Q}$-trees $T_{1}$ and $T_{2}$ are isomorphic. Since we can determine if two labeled $\mathcal{P} \mathcal{Q}$-trees $T_{1}$ and $T_{2}$ are isomorphic, the isomorphism of interval graphs can be determined in linear time.

$\mathcal{M P Q}$-trees, which stands for modified $\mathcal{P} \mathcal{Q}$-trees, are developed by Korte and Möhring to simplify the construction of $\mathcal{P} \mathcal{Q}$-trees [14].The $\mathcal{M P Q}$-tree $T^{*}$ assigns sets of vertices (possibly empty) to the nodes of a $\mathcal{P} \mathcal{Q}$-tree $T$ representing an interval graph $G=(V, E)$. A $\mathcal{P}$-node is assigned only one set, while a $\mathcal{Q}$-node has a set for each of its sons (ordered from left to right according to the ordering of the sons).

For a $\mathcal{P}$-node $\hat{P}$, this set consists of those vertices of $G$ contained in all maximal cliques represented by the subtree or $\hat{P}$ in $T$, but in no other cliques ${ }^{\dagger}$. For a $\mathcal{Q}$-node $\hat{Q}$, the definition is more involved. Let $Q_{1}, \cdots, Q_{m}$ $(m \geq 3)$ be the set of the sons (in consecutive order) of $\hat{Q}$, and let $T_{i}$ be the subtree of $T$ with root $Q_{i}$. We then assign a set $S_{i}$, called section, to $\hat{Q}$ for each $Q_{i}$. Section $S_{i}$ contains all vertices that are contained in all maximal cliques of $T_{i}$ and some other $T_{j}$, but not in any clique belonging to some other subtree of $T$ that is not below $\hat{Q}$.

In [14], Korte and Möhring showed two algorithms that construct an $\mathcal{M P} \mathcal{Q}$-tree for given interval graph. The first one constructs an $\mathcal{M P \mathcal { Q }}$-tree for given interval graph using its $\mathcal{P} \mathcal{Q}$-tree. The $\mathcal{M P \mathcal { Q }}$-tree directly corresponds to the labeled $\mathcal{P} \mathcal{Q}$-tree; the sets of vertices assigned in the $\mathcal{M P} \mathcal{Q}$-tree directly correspond to the "characteristic nodes" in [6]. Since the labeled $\mathcal{P} \mathcal{Q}$-tree is canonical, so is the constructed $\mathcal{M P} \mathcal{Q}$-tree. The second algorithm constructs an $\mathcal{M P \mathcal { Q }}$-tree from given interval graph directly without constructing $\mathcal{P} \mathcal{Q}$-trees in [3]. Although it does not shown explicitly, the $\mathcal{M P Q}$-tree constructed by the second algorithm is the same as the $\mathcal{M P} \mathcal{Q}$-tree by the first algorithm. Thus the graph isomorphism problem can be solved in linear time using the $\mathcal{M P} \mathcal{Q}$-trees, which can be obtained without constructing $\mathcal{P} \mathcal{Q}$-trees in [3].

The property of $\mathcal{M P Q}$-trees is summarized as follows [14, Theorem 2.1]:

Theorem 2 Let $T^{*}$ be the canonical $\mathcal{M P Q}$-tree for given interval graph $G=(V, E)$. Then

(a) $T^{*}$ can be obtained in $O(|V|+|E|)$ time and $O(|V|)$ space.

(b) Each maximal clique of $G$ corresponds to a path in $T^{*}$ from the root to a leaf, where each vertex $v \in V$ is as close as possible to the root.

\footnotetext{
$N$.

${ }^{\dagger}$ We will use $\hat{P}, \hat{Q}$, and $\hat{N}$ for describing a $\mathcal{P}$-node, $\mathcal{Q}$-node, any node, respectively to distinguish probe set $P$ and nonprobe set
} 


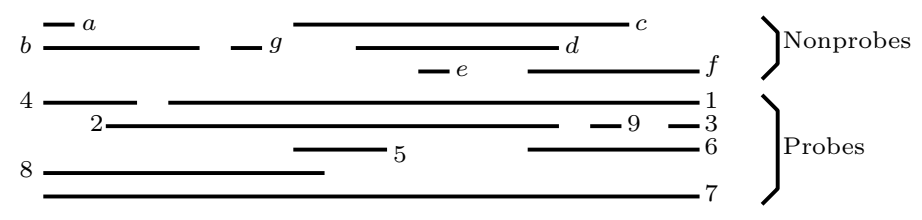

Figure 1: A given interval probe graph $G$

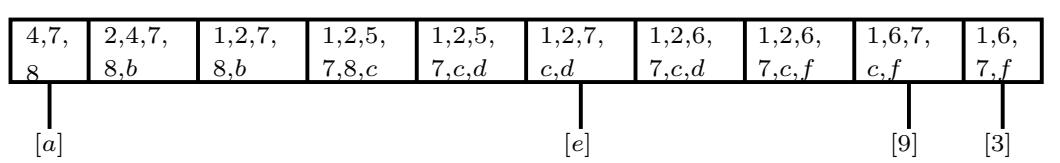

Figure 2: The $\mathcal{M P} \mathcal{Q}$-tree of $G-g$

(c) In $T^{*}$, each vertex $v$ appears in either one leaf, one $\mathcal{P}$-node, or consecutive sections $S_{i}, S_{i+1}, \cdots, S_{i+j}$ (with $j>0$ ) in a $\mathcal{Q}$-node.

(d) The root of $T^{*}$ contains all vertices belonging to all maximal cliques, while the leaves contain the simplicial vertices.

Proof. The claims (a) and (b) are stated in [14, Theorem 2.1]. The claim (c) is immediately obtained by the fact that the maximal cliques containing a fixed vertex occur consecutively in $T$; see [14] for further details. The claim (d) is also stated in [14, p. 71].

We note that there are no vertices that appear in only one section in a $\mathcal{Q}$-node (since such a vertex appears in the subtree of the section). Thus each vertex in a $\mathcal{Q}$-node appears at least two consecutive sections.

Lemma 3 Let $\hat{Q}$ be a $\mathcal{Q}$-node in the canonical $\mathcal{M P} \mathcal{Q}$-tree. Let $S_{1}, \cdots, S_{k}$ (in this order) be the sections of $\hat{Q}$, and let $U_{i}$ denote the set of vertices occurring below $S_{i}$ with $1 \leq i \leq k$. Then we have the following;

(a) $S_{i-1} \cap S_{i} \neq \emptyset$ for $2 \leq i \leq k$,

(b) $S_{1} \subseteq S_{2}$ and $S_{k} \subseteq S_{k-1}$,

(c) $U_{1} \neq \emptyset$ and $U_{k} \neq \emptyset$,

(d) $\left(S_{i} \cap S_{i+1}\right) \backslash S_{1} \neq \emptyset$ and $\left(S_{i-1} \cap S_{i}\right) \backslash S_{k} \neq \emptyset$ for $2 \leq i \leq k-1$,

(e) $S_{i-1} \neq S_{i}$ with $2 \leq i \leq k-1$, and

(f) $\left(S_{i-1} \cup U_{i-1}\right) \backslash S_{i} \neq \emptyset$ and $\left(S_{i} \cup U_{i}\right) \backslash S_{i-1} \neq \emptyset$ for $2 \leq i \leq k$.

Proof. The results in [14] lead us from (a) to (e) immediately. Thus we show (f). If $\left(S_{i-1} \cup U_{i-1}\right) \backslash S_{i}=\emptyset$, we have $U_{i-1}=\emptyset$ and $S_{i-1} \subset S_{i}$. In the case, $S_{i-1}$ is redundant section; we can obtain more compact $\mathcal{M P \mathcal { P }}$-tree by removing $S_{i-1}$. This fact contradicts that the $\mathcal{M P \mathcal { Q }}$-tree is canonical. Thus (f) is settled.

For a given enhanced interval probe graph $G^{+}=\left(P, N, E \cup E^{+}\right)$, let $u$ and $v$ be any two nonprobes with $\{u, v\} \notin E^{+}$. Then, we say that $u$ intersects $v$ if $I_{u} \cap I_{v} \neq \emptyset$ for all affirmative interval graphs of $G^{+}$. The nonprobes $u$ and $v$ are independent if $I_{u} \cap I_{v}=\emptyset$ for all affirmative interval graphs of $G^{+}$. Otherwise, we say that the nonprobe $u$ potentially intersects $v$. Intuitively, if $u$ potentially intersects $v$, we cannot determine their relation without experiments.

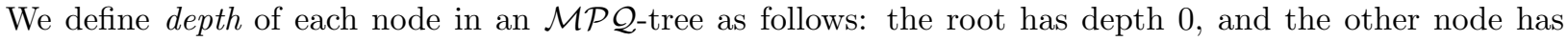
depth $d+1$, where $d$ is the depth of its parent. We also define depth of a vertex in $G$ by the depth of the node in the $\mathcal{M P} \mathcal{Q}$-tree that contains $v$. By Theorem 2(c), the depth of a vertex $v$ in the $\mathcal{M P} \mathcal{Q}$-tree is uniquely determined, and we denote by $\operatorname{dep}(v)$. 

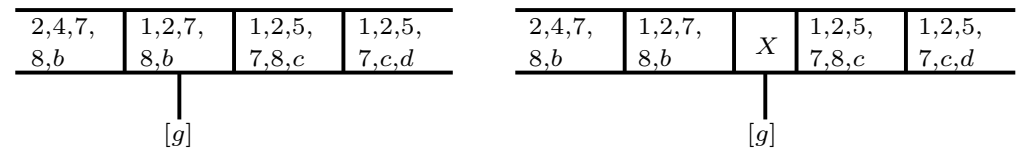

Figure 3: Four $\mathcal{M P \mathcal { Q }}$-trees of $G$

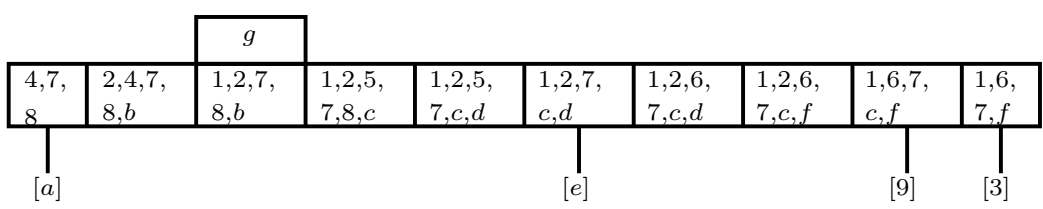

Figure 4: The extended $\mathcal{M P} \mathcal{Q}$-tree of $G$

\section{$2.3 \quad$ Extended $\mathcal{M P \mathcal { Q } \text { -trees }}$}

If given graph is an interval graph, the corresponding $\mathcal{M P Q}$-tree is uniquely determined up to isomorphism. However, for an interval probe graph, this is not in the case. For example, consider an interval probe graph $G=(P, N, E)$ with $P=\{1,2,3,4,5,6,7,8,9\}$ and $N=\{a, b, c, d, e, f, g\}$ given in Figure 1. If the graph does not contain the nonprobe $g$, we have the canonical $\mathcal{M P \mathcal { P }}$-tree in Figure 2. However, the graph is an interval probe graph and we do not know if $g$ intersects $b$ and/or $c$ since they are nonprobes. According to the relations

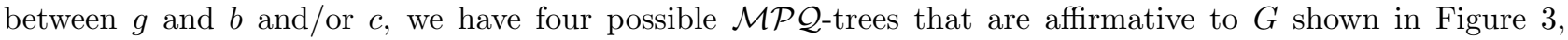
where $X$ is either $\{1,2,7,8\},\{1,2,7,8, c\}$, or $\{1,2,7,8, b, c\}$. We call such a vertex $g$ floating leaf (later, it will be shown that such a vertex has to be a leaf in an $\mathcal{M P Q}$-tree). For a floating leaf, there is a corresponding $\mathcal{Q}$-node (which also will be shown later). Thus we extend the notion of a $\mathcal{Q}$-node to contain the information of the floating leaf. A floating leaf appears consecutive sections of a $\mathcal{Q}$-node $\hat{Q}$ as the ordinary vertices in $\hat{Q}$. To distinguish them, we draw them over the corresponding sections; see Figure 4. Further details will be discussed in Section 3.

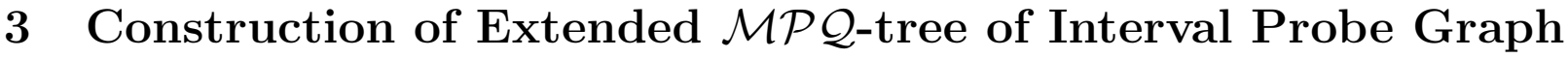

Let $G=(P, N, E)$ be a given interval probe graph, and $G^{+}=\left(P, N, E \cup E^{+}\right)$be the corresponding enhanced interval probe graph, where $E^{+}$is the set of enhanced edges. In our algorithm, simplicial nonprobes play an important role; we partition the set $N$ of nonprobes to two sets $N^{*}$ and $N_{S}$ defined as follows;

$$
\begin{aligned}
& N_{S}=\left\{u \mid u \text { is simplicial in } G^{+}\right\}, \\
& N^{*}=N \backslash N_{S} .
\end{aligned}
$$

For example, for the graph $G=(P, N, E)$ in Figure $1, E^{+}=\{\{c, d\},\{c, f\}\}, N_{S}=\{a, e, g\}$, and $N^{*}=$ $\{b, c, d, f\}$. Then the outline of the algorithm is as follows.

A0. For a given interval probe graph $G=(P, N, E)$, compute the enhanced interval probe graph $G^{+}=$ $\left(P, N, E \cup E^{+}\right)$

A1. Partition $N$ into two subsets $N^{*}$ and $N_{S}$;

A2. Construct the $\mathcal{M P Q}$-tree $T^{*}$ of $G^{*}=\left(P, N^{*}, E^{*}\right)$, where $E^{*}$ is the set of edges induced by $P \cup N^{*}$ from $G^{+}$

A3. Embed each nonprobe $v$ in $N_{S}$ into $T^{*}$.

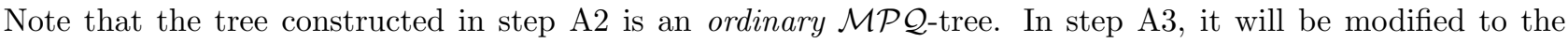
extended $\mathcal{M P \mathcal { Q }}$-tree. The following observation is obtained by definition:

Observation 4 Let $v$ be a nonprobe in $N_{S}$. Then for any two vertices $u_{1}, u_{2} \in N_{G^{+}}(v), I_{u_{1}} \cap I_{u_{2}} \neq \emptyset$.

It is also easy to see that if $G=(P, N, E)$ is connected, so is $G^{*}$. 


\begin{tabular}{|l|l|l|l|l|l|l|l|}
\hline 2,7, & $1,2,7$, & $1,2,5$, & $1,2,5$, & $1,2,6$, & $1,2,6$, & $1,6,7$, & 1,6, \\
$8, b$ & $8, b$ & $7,8, c$ & $7, c, d$ & $7, c, d$ & $7, c, f$ & $c, f$ & $7, f$ \\
\hline
\end{tabular}

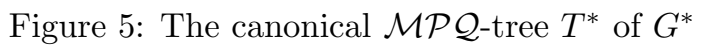

\subsection{Construction of $\mathcal{M P Q}$-tree of $G^{*}$}

Let $G^{*}=\left(P, N^{*}, E^{*}\right)$ be the enhanced interval probe graph induced by $P$ and $N^{*}$. The following lemma plays an important role in this subsection.

Lemma 5 Let $u$ and $v$ be any nonprobes in $N^{*}$. Then there is an interval representation of $G^{*}$ such that $I_{u} \cap I_{v} \neq \emptyset$ if and only if $\{u, v\} \in E^{+}$.

Proof. If $\{u, v\} \in E^{+}, I_{u} \cap I_{v} \neq \emptyset$ by definition. Thus we assume that $\{u, v\} \notin E^{+}$, and show that there is an interval representation of $G^{*}$ such that $I_{u} \cap I_{v}=\emptyset$. We fix an interval representation of $G^{*}$, and assume that $I_{u} \cap I_{v} \neq \emptyset$. When $N(u) \cap N(v)=\emptyset$, it is easy to modify to satisfy $I_{u} \cap I_{v}=\emptyset$. Thus we assume that $N(u) \cap N(v) \neq \emptyset$. We first show that $N(u) \nsubseteq N(v)$ and $N(v) \nsubseteq N(u)$. If $N(u) \subseteq N(v)$, since $\{u, v\} \notin E^{+}$, all vertices in $N(u)$ intersect with each other. Thus, $N(u)$ induces a clique, which contradicts $u \in N^{*}$. Hence $N(u) \nsubseteq N(v)$ and $N(v) \nsubseteq N(u)$. Without loss of generality, we can assume that $L(u)<L(v)<R(u)<R(v)$. Let $w_{1}$ and $w_{2}$ are any probes which intersect the interval $[L(v), R(u)]$. Then, since $\{u, v\} \notin E^{+}, I_{w_{1}} \cap I_{w_{2}} \neq \emptyset$. Thus, by the Helly property (see, e.g.,[1]), there is a point $p$ in the interval $[L(v), R(u)]$ such that all probes contain $p$. We replace the point $p$ in all intervals by a small interval $[p-\epsilon, p+\epsilon]$, and then we replace $I_{u}$ by $[L(u), p-\epsilon]$ and $I_{v}$ by $[p+\epsilon, R(v)]$. The replacement has no effect to the relations between $u$ (or $v$ ) and probes. We here show that the replacement also has no effect to the relations between $u$ (or $v$ ) and other nonprobes. To derive contradictions, we assume hat the relation between $u$ and a nonprobe $w$ is changed. Since the interval $I_{u}$ is shortened, $w \in N(u)$ becomes $w \notin N(u)$ by the replacement. Since both of $u$ and $w$ are nonprobes, there are two independent probes $t_{1}$ and $t_{2}$ that guarantee $w \in N(u)$. Then, replacing $[L(u), R(u)]$ by $[L(u), p-\epsilon]$, at least one of $t_{1}$ and $t_{2}$, say $t$, changes from $t \in N(u)$ to $t \notin N(u)$. However this contradicts the definition of the point $p$, which should be contained in $t$, and we have $t \in N(u)$ after replacement. Thus the replacement has no effect to the relations between $u$ (or $v$ ) and other nonprobes. Hence we obtain a new valid interval representation of $G^{*}$ with $I_{u} \cap I_{v}=\emptyset$. Repeating this process for each pair we have the lemma.

The definition of (enhanced) interval probe graphs and Lemma 5 imply the main theorem in this section:

Theorem 6 The enhanced interval probe graph $G^{*}=\left(P, N^{*}, E^{*}\right)$ is an interval graph.

Hereafter we call the graph $G^{*}=\left(P, N^{*}, E^{*}\right)$ the backbone interval graph of $G^{+}=\left(P, N, E \cup E^{+}\right)$. For any given interval graph, its corresponding $\mathcal{M P Q}$-tree can be computed in linear time [14]. Thus we also have the following corollary:

Corollary 7 The canonical $\mathcal{M P} \mathcal{Q}$-tree $T^{*}$ of $G^{*}$ can be computed in linear time.

Thus the step A2 is rewritten as follows;

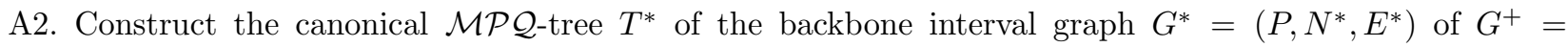
$\left(P, N, E \cup E^{+}\right)$;

In the canonical $\mathcal{M P Q}$-tree $T^{*}$, for each pair of nonprobes $u$ and $v$, their corresponding intervals intersect if and only if $\{u, v\} \in E^{+}$. This implies the following observation.

Observation 8 The canonical $\mathcal{M P} \mathcal{Q}$-tree $T^{*}$ gives us the possible interval representations of $G^{*}$ such that two nonprobes in $N^{*}$ do not intersect as possible as they can.

For example, for the graph $G=(P, N, E)$ in Figure 1, the canonical $\mathcal{M P} \mathcal{Q}$-tree of the backbone interval graph

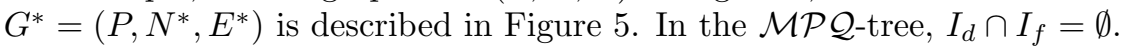

We note that in $T^{*}$, with suitable labels, we can distinguish nonprobes from probes, and nonprobes in $N^{*}$ from nonprobes in $N_{S}$, which will be added later. Now, our main task is that embedding each vertex in $N_{S}$ into the canonical $\mathcal{M P} \mathcal{Q}$-tree $T^{*}$ without breaking canonicality. 


\subsection{Embedding of Nonprobes in $N_{S}$}

We first show two lemmas for the nonprobes in $N_{S}$.

Lemma 9 For each nonprobe $v$ in $N_{S}$, all vertices in $N(v)$ are probes.

Proof. To derive a contradiction, we assume that a nonprobe $v^{\prime}$ is in $N(v)$. Then $\left\{v, v^{\prime}\right\}$ is in $E^{+}$. Thus there are two probes $u$ and $u^{\prime}$ such that $\{u, v\},\left\{u, v^{\prime}\right\},\left\{u^{\prime}, v\right\}$, and $\left\{u^{\prime}, v^{\prime}\right\}$ are in $E$, and $\left\{u, u^{\prime}\right\}$ is not in $E$, which contradicts Observation 4.

Lemma 10 For any interval probe graph $G$, there is an affirmative interval graph $G^{\prime}$ such that every nonprobe $v$ in $N_{S}$ of $G$ is also simplicial in $G^{\prime}$.

Proof. Let $v$ be any nonprobe in $N_{S}$ such that $v$ is not simplicial in $G^{\prime}$. By the Helly property, there is a point $p$ such that all probes in $N(v)$ contains $p$. We replace the point $p$ in all intervals by a small interval $[p-\epsilon, p+\epsilon]$, and we set $R(v)=L(v)=p$. Then $v$ is simplicial in the interval graph corresponding to the new interval representation, and the interval graph is still affirmative. Thus, repeating this process, we have the lemma.

By Lemma 10 and Theorem 2(d), we have the following corollary.

Corollary 11 For any interval probe graph $G$, there is an affirmative interval graph $G^{\prime}$ such that every nonprobe $v$ in $N_{S}$ of $G$ is in a leaf of the $\mathcal{M P Q}$-tree of $G^{\prime}$.

Our embedding is an extension of the embedding by Korte and Möhring [14] to deal with nonprobes. Each node $\hat{N}$ (including $\mathcal{Q}$-node) of the current tree $T^{*}$ and each section $S$ of a $\mathcal{Q}$-node is labeled according to how the nonprobe $v$ in $N_{S}$ is related to the probes in $\hat{N}$ or $S$. Nonprobes in $\hat{N}$ or $S$ are ignored. The label is $\infty$, 1 , or 0 if $v$ is adjacent to all, some, or no probe from $\hat{N}$, or $S$, respectively. Empty sets (or the sets containing only nonprobes) obtain the label 0 . Labels 1 and $\infty$ are called positive labels.

Lemma 12 For a nonprobe $v$ in $N_{S}$, all nodes with a positive label are contained in a unique path of $T^{*}$.

Proof. By definition, $v$ is simplicial, or $N(v)$ induce a clique. Thus Theorem 2(b) implies the lemma.

Let $\mathrm{P}^{\prime}$ be the unique minimal path in $T^{*}$ containing all nodes with positive label. Let $\mathrm{P}$ be a path from the

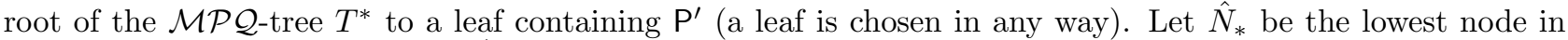
$\mathrm{P}$ with positive label. (That is, $\hat{N}_{*}$ is the node of the largest depth in $\mathrm{P}^{\prime}$.) If $\mathrm{P}$ contains nonempty $\mathcal{P}$-nodes or sections above $\hat{N}_{*}$ with label 0 or 1 , let $\hat{N}^{*}$ be the highest such $\mathcal{P}$-node or $\mathcal{Q}$-node containing the section. Otherwise put $\hat{N}_{*}=\hat{N}^{*}$.

When $\hat{N}_{*} \neq \hat{N}^{*}$, we have the following lemma:

Lemma 13 We assume that $\hat{N}_{*} \neq \hat{N}^{*}$. Let $\hat{Q}$ be any $\mathcal{Q}$-node with sections $S_{1}, \cdots, S_{k}$ in this order between $\hat{N}_{*}$ and $\hat{N}^{*}$. If $\hat{Q}$ is not $\hat{N}^{*}$, all neighbors of $v$ in $\hat{Q}$ appear in either $S_{1}$ or $S_{k}$.

Proof. We first observe that $\hat{N}^{*}$ contains at least one probe $w$ of $v$ with $w \notin N(v)$ since $\hat{N}^{*}$ is non-empty and the label of $\hat{N}^{*}$ is 0 or 1 . We assume that $v$ has a neighbor $u$ in $Q$ with $u \notin S_{1}$ and $u \notin S_{k}$ to derive a contradiction. Let $U_{1}$ and $U_{k}$ be the set of vertices occurring below $S_{1}$ and $S_{k}$, respectively. By Lemma 3(c), $U_{1} \neq \emptyset$ and $U_{k} \neq \emptyset$. Thus there are two vertices $u_{1} \in U_{1}$ and $u_{k} \in U_{k}$ such that $I_{u_{1}} \subseteq I_{w}, I_{u} \subseteq I_{w}, I_{u_{k}} \subseteq I_{w}$, and $R\left(I_{u_{1}}\right)<L\left(I_{u}\right)<R\left(I_{u}\right)<L\left(u_{k}\right)$ (or $R\left(I_{u_{k}}\right)<L\left(I_{u}\right)<R\left(I_{u}\right)<L\left(u_{1}\right)$ ). Thus we have $I_{u} \subset I_{w}$, which contradicts that $w \notin N(v)$ and $u \in N(v)$.

Note that Lemmas 12 and 13 correspond to [14, Lemma 4.1]. However, Lemma 13 does not hold at the node $\hat{N}^{*}$. We are now ready to use the bottom-up strategy from $\hat{N}_{*}$ to $\hat{N}^{*}$ as in [14]. In [14], the ordering of vertices are determined by LexBFS. In our algorithm, the step A3 consists of the following substeps;

A3.1. while there is a nonprobe $v$ such that $\hat{N}_{*} \neq \hat{N}^{*}$ for $v$, embed $v$ into $T^{*}$;

A3.2. while there is a nonprobe $v$ such that $\hat{N}_{*}=\hat{N}^{*}$ for $v$ and $v$ is not a floating leaf, embed $v$ into $T^{*}$;

A3.3. embed each nonprobe $v$ (such that $\hat{N}_{*}=\hat{N}^{*}$ for $v$ and $v$ is a floating leaf) into $T^{*}$.

As shown later, an embedding of a nonprobe $v$ with $\hat{N}_{*} \neq \hat{N}^{*}$ merges some nodes into one new $\mathcal{Q}$-node. Thus, during step A3.1, embedding of a nonprobe $v$ can change the condition of other nonprobes $u$ from " $\hat{N}_{*} \neq \hat{N}^{*}$ " to " $\hat{N}_{*}=\hat{N}^{*} "$. We note that A3.1 and A3.2 do not generate floating leaves, and all floating leaves are embedded 


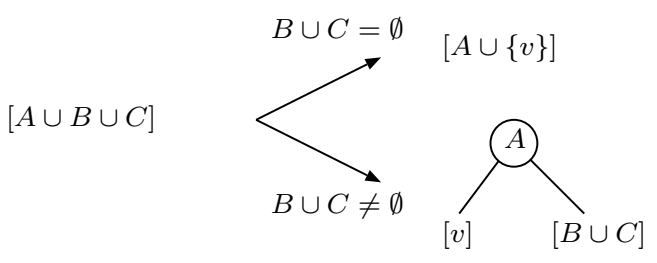

Figure 6: Template L1 when $\hat{N}^{*}=\hat{N}_{*}$ is a leaf
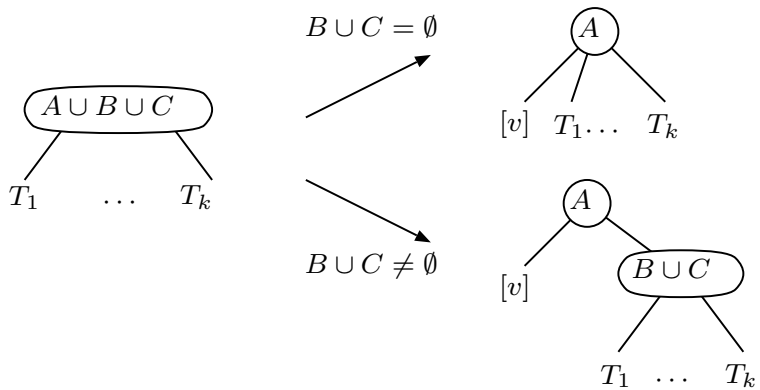

Figure 7: Template P1 when $\hat{N}^{*}=\hat{N}_{*}$ is a $\mathcal{P}$-node

in step A3.3, which will be shown later. Hence the templates used in steps A3.1 and A3.2 are not required to manage floating leaves.

Hereafter, we suppose that the algorithm picks up some nonprobe $v$ from $N_{S}$ and it is going to embed $v$ into $T^{*}$. In most cases, the vertex set $V_{N}$ of the current node or section is partitioned into $A, B$, and $C$ defined as follows;

$$
\begin{aligned}
A & :=P \cap V_{N} \cap N(v), \\
B & :=\left(P \cap V_{N}\right) \backslash A, \\
C & :=N \cap V_{N} .
\end{aligned}
$$

Since we extend the templates in [14], we use the same names of templates as L1, P2, and so on, which is an extension of the corresponding templates in [14] (templates from Q4 to Q7 are new templates). We also use the help templates H1 and H2 in [14] if they can be applied; it is simple and omitted here. Through the embedding, we keep the following assertion:

Assertion 14 (1) Each nonprobe in $N_{S}$ has no intersection with unnecessary nonprobes,

(2) each leaf contains either vertices in $P \cup N^{*}$ or one nonprobe in $N_{S}$, and

(3) each nonprobe in $N_{S}$ is in a leaf.

\subsubsection{Templates for the nonprobe with $\hat{N}_{*}=\hat{N}^{*}$}

We first assume that $\hat{N}^{*}=\hat{N}_{*}$, which occurs in steps A3.2 and A3.3. If the node is a leaf or a $\mathcal{P}$-node, we use template L1 in Figure 6 or P1 in Figure 7, respectively. If $\hat{N}^{*}=\hat{N}_{*}$ is a $\mathcal{Q}$-node with sections $S_{1}, \cdots, S_{k}$ in this order, $v$ can be a floating leaf. We let $A:=\left(\cup_{1 \leq i \leq k} S_{i}\right) \cap N(v)$. Let $\ell$ be the minimum index with $A \subseteq S_{\ell}$ and $r$ be the maximum index with $A \subseteq S_{r}$. That is, $A \nsubseteq \subseteq S_{i}$ for each $i<\ell$ and $i>r$, and $A \subseteq S_{j}$ for each $\ell \leq j \leq r$. Then there are four cases:

(a) $\ell=1$ and $A \subset S_{\ell} \cap P$. In the case, $v$ may be a leaf of a new section $S_{0}:=A \subset S_{1}$. The case $r=k$ and $A \subset S_{k} \cap P$ is symmetric.

(b) $A=S_{j} \cap P$ for some $\ell \leq j \leq r$. In the case, $v$ may be a leaf under the section $S_{j}$.

(c) $A=S_{j} \cap S_{j+1} \cap P$ for some $\ell \leq j<r$. In the case, $v$ may be a leaf under the new section $S$ between $S_{j}$ and $S_{j+1}$ with $S:=A \cup\left(S_{j} \cap S_{j+1} \cap N\right)$. 


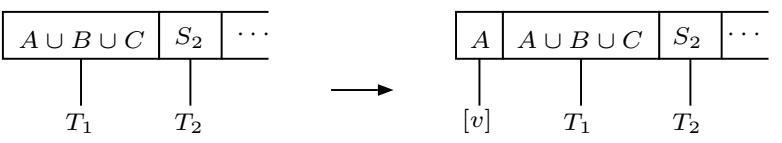

Figure 8: Template Q2 for (1) $\hat{N}_{*}=\hat{N}^{*}$ and $A \subset S_{1} \cap P$, or (2) $\hat{N}=\hat{N}_{*} \neq \hat{N}^{*}, A \subseteq S_{1}$, and $A \nsubseteq \cap_{1 \leq i \leq k} S_{i}$ (Q6-1)
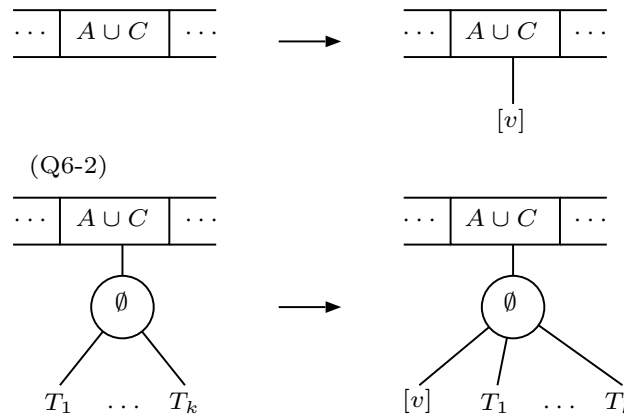
$(\mathrm{Q} 6-3)$

Figure 9: Template Q6 for $\hat{N}_{*}=\hat{N}^{*}$ and $A=S_{j} \cap P$ for some $\ell \leq j \leq r$

(d) $S_{j} \cap S_{j+1} \cap P \subset A \subset S_{j} \cap P$ or $S_{j} \cap S_{j+1} \cap P \subset A \subset S_{j+1} \cap P$ for some $\ell \leq j<r$. In the case, $v$ may be a leaf under the new section $S$ between $S_{j}$ and $S_{j+1}$ with $S:=A \cup\left(S_{j} \cap S_{j+1} \cap N\right)$.

The algorithm checks if the position of the $v$ is uniquely determined. If it is uniquely determined, the algorithm embeds $v$ into the place in step A3.2. If exactly one of the cases (a) to (d) occurs, we use the templates as follows. In the case (a), template Q2 in Figure 8 is used. In the case (b), we use three templates Q6-1, Q6-2, and Q6-3 in Figure 9 as follows; if the section $S_{j}$ has no child, template Q6-1 is used and $v$ is added as a leaf under $S_{j}$; if the root of the subtree under $S_{j}$ is a $\mathcal{P}$-node with empty label, template Q6-2 is used and $v$ is added as a leaf under the $\mathcal{P}$-node; or otherwise, template Q6-3 is used and $v$ is added as a leaf under a new $\mathcal{P}$-node with empty label under $S_{j}$. We note that Assertion 14(2) holds if $\hat{R}$ contains nonprobes. In the case (c) or (d), template Q7 in Figure 8 is used; we note that we have $A \cup\left(S_{j} \cap S_{j+1} \cap N\right)=S_{j} \cap S_{j+1}$ in the case (c). We have one more case that the position of the $v$ may be uniquely determined; $\ell=1, r=k$, and $\left(S_{i} \cap S_{i+1} \cap P\right) \backslash A \neq \emptyset$ for each $1 \leq i<k$. In the case, we use the template Q1-1 in Figure 11. In Figure 11, for each $1 \leq i \leq k, B_{i}:=\left(S_{i} \cap P\right) \backslash N(v)$ and $C_{i}$ denotes nonprobes in $S_{i}$. We note that $B_{i} \cap B_{i+1} \neq \emptyset$ for each $1 \leq i<k$; otherwise, $v$ can be a floating leaf under the section between $S_{i}$ and $S_{i+1}$.

If the position is not uniquely determined, $v$ is a floating leaf. Thus, in the case, the embedding is postponed until step A3.3. Then we use template Q4 in Figure 12 for such $\ell$ and $r$; in the figure, $R_{i}$ denotes the set of floating leaves in $S_{i}$. Hereafter, we assume that each section $S_{i}$ between $R_{\ell}$ and $R_{r}$ knows if the vertex $v$ can be a floating leaf "under $S_{i}$ ", "right of $S_{i}$ ", and "left of $S_{i}$ "; that is, if $v$ can be a leaf $[v]$ under $S$ between $S_{i}$ and $S_{i+1}$, they know that $v$ can be a floating leaf at the left side of $S_{i}$, and the right side of $S_{i+1}$, respectively. If $v$ can be a floating leaf under such sections (including non-existent sections), we say $v$ can hang down the section.

We have the following observation.

Observation 15 In steps A3.2 and A3.3, all $\mathcal{Q}$-nodes are neither divided nor merged.

\subsubsection{Templates for the nonprobe with $\hat{N}_{*} \neq \hat{N}^{*}$}

When $\hat{N}_{*} \neq \hat{N}^{*}$, we use the same bottom-up strategy from $\hat{N}_{*}$ to $\hat{N}^{*}$ as in [14]. Let $\hat{N}$ denote the current node that starts from $\hat{N}_{*}$ and ends up at $\hat{N}^{*}$. The algorithm consists of three phases; (1) $\hat{N}=\hat{N}_{*},(2) \hat{N}_{*} \hat{N}_{*}$

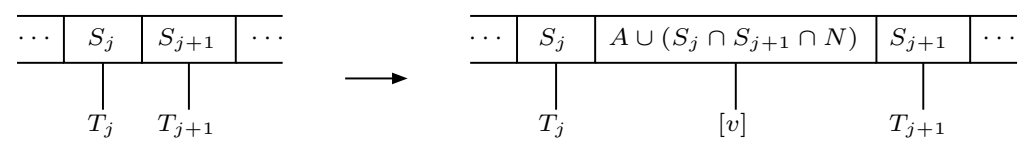

Figure 10: Template Q7 for $\hat{N}_{*}=\hat{N}^{*}$ and $S_{j} \cap S_{j+1} \cap P \subset A \subseteq S_{j+1} \cap P$ or $S_{j} \cap S_{j+1} \cap P \subset A \subseteq S_{j} \cap P$ 

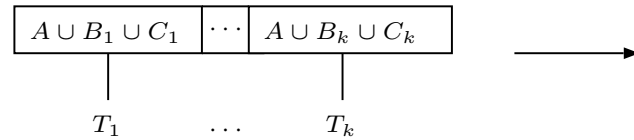

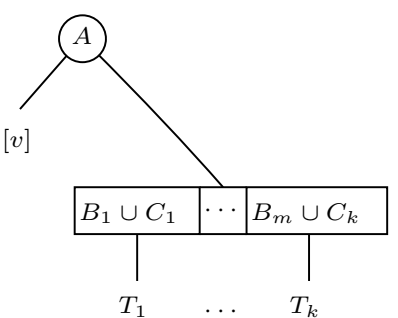

Figure 11: Template Q1-1
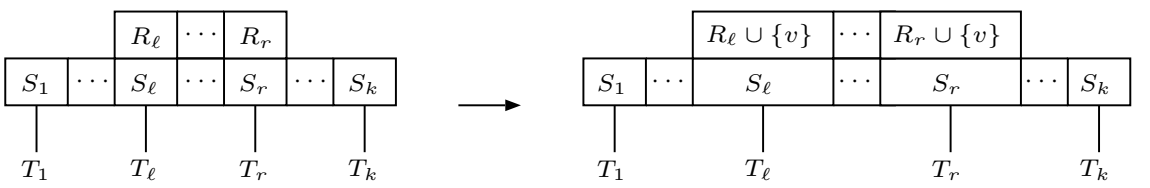

Figure 12: Template Q4

and $\hat{N} \neq \hat{N}^{*}$, and (3) $\hat{N}=\hat{N}^{*}$. The first two phases are the natural extension of the templates in [14] by Lemmas 12 and 13 which correspond to [14, Lemma 4.1]. However, the algorithm uses one more template in the third phase, since Lemma 13 does not hold. The templates in the case $\hat{N}_{*} \neq \hat{N}^{*}$ never generate floating leaves. Therefore, since they are applied in step A3.1, the templates in the case are not required to manage floating leaves.

(1) $\hat{N}=\hat{N}_{*} \neq \hat{N}^{*}$. Since the label of $\hat{N}=\hat{N}_{*}$ is positive, $A:=\hat{N} \cap N(v) \neq \emptyset$. If $\hat{N}$ is a leaf or a $\mathcal{P}$-node, the algorithm uses template L2 in Figure 13 or P2 in Figure 14, respectively. When $\hat{N}$ is a $\mathcal{Q}$-node, we can use Lemmas 12 and 13 in this case. Thus we have two subcases, which correspond to templates Q1 and Q2 in [14]. By Lemma 13, we assume that $A \subseteq S_{1}$ without loss of generality. The algorithm uses template Q1-2 in Figure 15 if $A \subseteq S_{k}$, and otherwise, it uses template Q2 in Figure 8 .

Observation 16 In any case, $v$ becomes a leaf $[v]$ under a non-empty section $S_{1}$ of a $\mathcal{Q}$-node since $A \neq \emptyset$.

(2) $\hat{N} \neq \hat{N}_{*}$ and $\hat{N} \neq \hat{N}^{*}$. If $\hat{N}$ is a $\mathcal{P}$-node, the algorithm uses template P3 in Figure 16. If $\hat{N}$ is a $\mathcal{Q}$-node, we can use Lemmas 12 and 13 again and the algorithm uses template Q3 in Figure 17. By a simple induction of the length of the path $\mathrm{P}$ with Observation 16, we again have the following observation (since $S_{1} \neq \emptyset$ in Figures 16 and 17$)$ :

Observation 17 In any case, $v$ becomes a leaf $[v]$ under a non-empty section $S_{1}$ of a $\mathcal{Q}$-node.

(3) $\hat{N}=\hat{N}^{*} \neq \hat{N}_{*}$. If $\hat{N}$ is a $\mathcal{P}$-node, the algorithm uses the template P3 in Figure 16 again. If $\hat{N}$ is a $\mathcal{Q}$-node, we cannot use Lemmas 13. Let $S_{i}^{\prime}$ be the section in $\hat{N}$ such that the subtree $T_{i}^{\prime}$ contains [ $\left.v\right]$. If $S_{i}^{\prime}$ is the leftmost

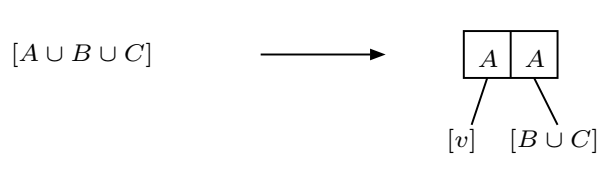

Figure 13: Template L2 for $\hat{N}=\hat{N}_{*} \neq \hat{N}^{*}$

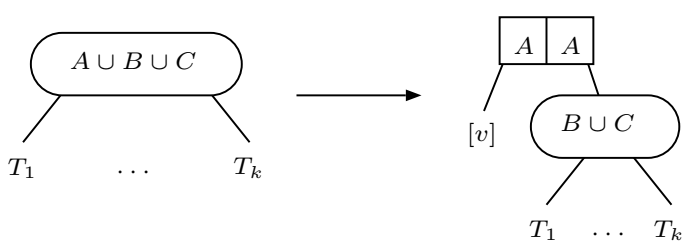

Figure 14: Template P2 for $\hat{N}=\hat{N}_{*} \neq \hat{N}^{*}$ 

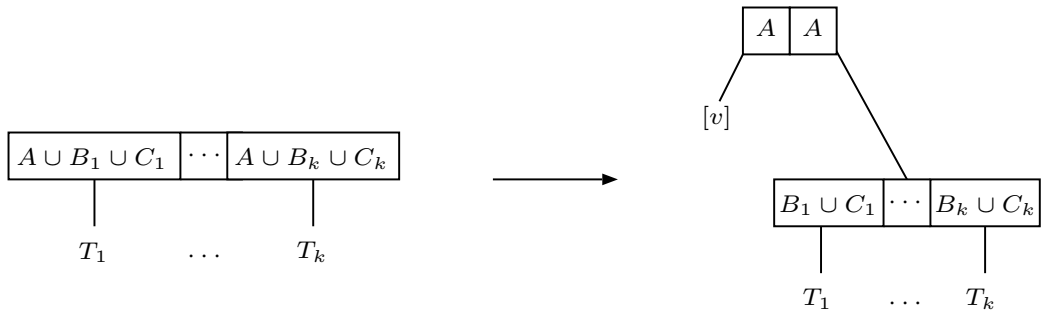

Figure 15: Template Q1-2 for $\hat{N}=\hat{N}_{*} \neq \hat{N}^{*}$ and $A \subseteq \cap_{1 \leq i \leq k} S_{i}$

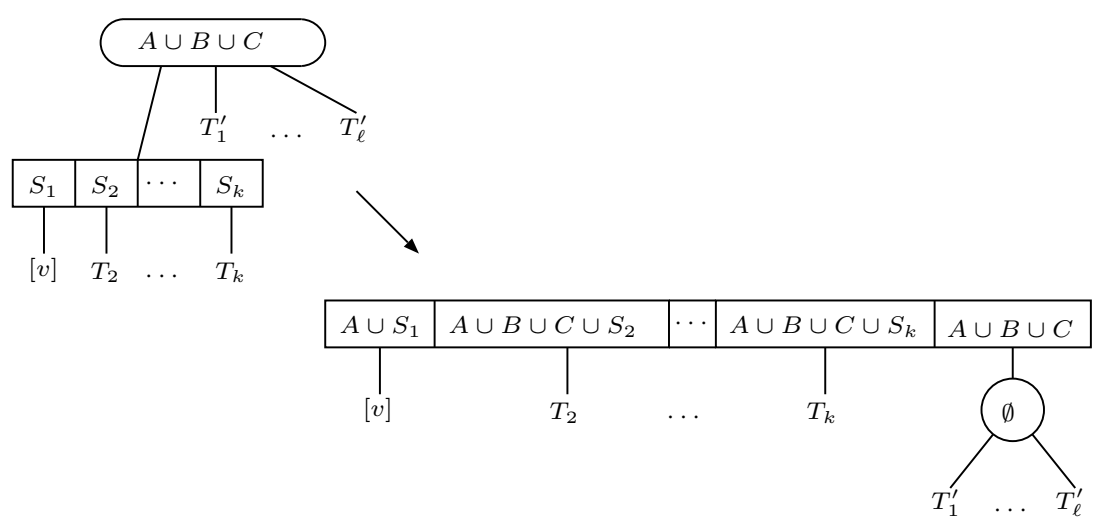

Figure 16: Template P3 for $\hat{N} \neq \hat{N}_{*}$ and $\hat{N} \neq \hat{N}^{*}$

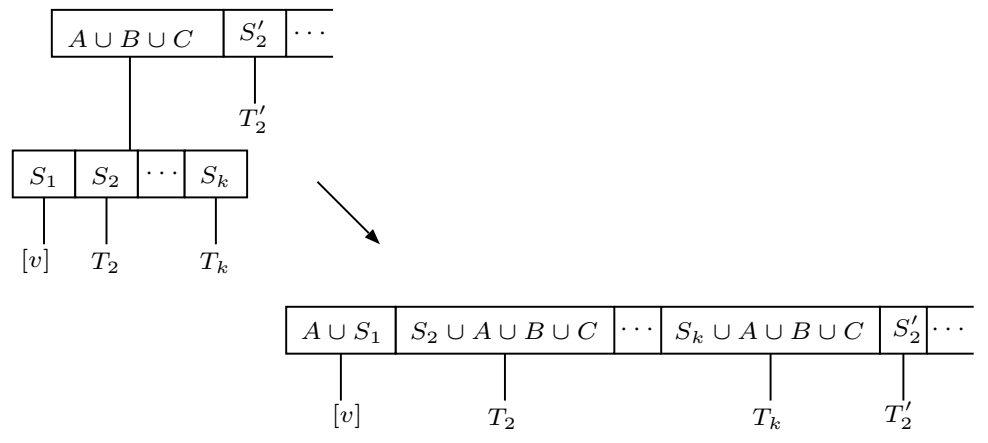

Figure 17: Template Q3 for $\hat{N} \neq \hat{N}_{*}$ and $\hat{N} \neq \hat{N}^{*}$ 


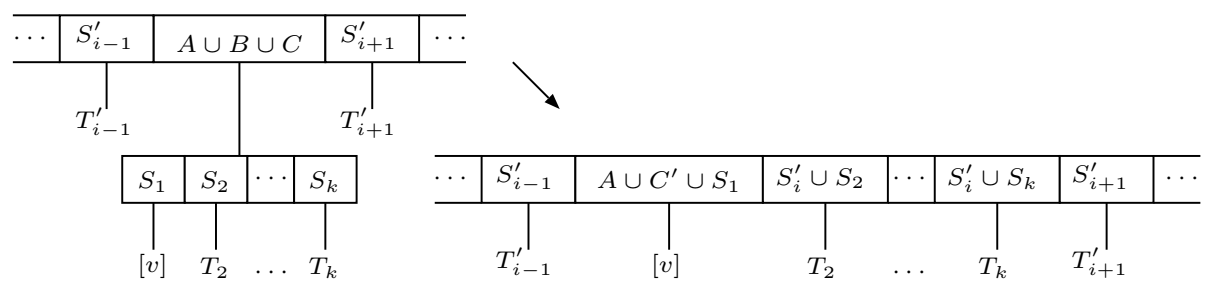

Figure 18: Template Q5 for $\hat{N}=\hat{N}^{*} \neq \hat{N}_{*}$ and $B \subseteq S_{i+1}^{\prime}$

or rightmost section in $\hat{N}$, we can use the template Q3 in Figure 17 again. Thus we assume that $1<i<k^{\prime}$, where $k^{\prime}$ is the number of sections in the $\mathcal{Q}$-node $\hat{N}$. Let $S_{i-1}^{\prime}$ and $S_{i+1}^{\prime}$ be the left and right sections of $S_{i}^{\prime}$, respectively. We now define $A:=N(v) \cap S_{i}^{\prime}$ and $B:=\left(S_{i}^{\prime} \cap P\right) \backslash A$. Then, since the label of $S_{i}^{\prime}$ is 0 or 1 , we have $B \neq \emptyset$. For the set $B$, we have the following lemma:

Lemma 18 Either $B \subseteq S_{i+1}^{\prime} \backslash S_{i-1}^{\prime}$ or $B \subseteq S_{i-1}^{\prime} \backslash S_{i+1}^{\prime}$.

Proof. Let $u$ be any vertex in $B$. By theorem $2(\mathrm{c}), u \in S_{i-1}^{\prime}$ or $u \in S_{i+1}^{\prime}$. Since $B \neq \emptyset, S_{i-1}^{\prime} \cap B=S_{i+1}^{\prime} \cap B=\emptyset$ does not occur. Thus it is sufficient to show that $S_{i-1}^{\prime} \cap B \neq \emptyset$ and $S_{i+1}^{\prime} \cap B \neq \emptyset$ implies a contradiction. We assume that there are two vertices $u_{1}$ and $u_{2}$ such that $u_{1} \in S_{i-1}^{\prime} \cap B$ and $u_{2} \in S_{i+1}^{\prime} \cap B$. Then $[v]$ cannot be a leaf under any sections $S_{i-1}^{\prime}, S_{i}^{\prime}$, and $S_{i+1}^{\prime}$. This implies that the graph $G$ is not an interval probe graph, which is a contradiction.

Without loss of generality, we assume that Lemma 18(a) occurs. That is, all vertices in $B$ appear from the section $S_{i}^{\prime}$ to the some sections on the right side of $S_{i}^{\prime}$. Let $C^{\prime}:=S_{i-1}^{\prime} \cap S_{i}^{\prime} \cap N$. That is, $C^{\prime}$ is the set of nonprobes appearing both of $S_{i-1}^{\prime}$ and $S_{i}$. Then we use template Q5 in Figure 18. In the figure, $C$ denotes the nonprobes in $S_{i}^{\prime}$; that is, $S_{i}^{\prime}=A \cup B \cup C$. We note that $C^{\prime} \subseteq C$, and Assertion 14(1) holds.

Example 19 For the graph $G=(P, N, E)$ in Figure 1 with its backbone interval graph in Figure 5, the extended $\mathcal{M P Q}$-tree $\tilde{T}$ is shown in Figure 4. The algorithm uses templates L2 and Q3 to embed a, and uses template Q4 to embed $g$ since it is a floating leaf. For the nonprobe $e$, only the case (c) in Section 3.2 .1 can be applied; $\{1,2,7,8, c, d\} \cap\{1,2,6,7, c, d\} \cap P=\{1,2,7\}=N(e)$. Thus its position is uniquely determined, and embedded between the sections. Note that we can know that $e$ intersects both of $c$ and $d$ with neither experiments nor enhanced edges. We also note that $I_{a}$ and $I_{b}$ could have intersection, but they are standardized according to Assertion 14(1).

\subsection{Analysis of Algorithm}

\section{Correctness}

Since the correctness of steps A0, A1, and A2 follows from Theorem 6, we concentrate on step A3. First, the templates cover all formally distinct cases. All templates for the case $\hat{N}_{*}=\hat{N}^{*}$ with the help-templates H1 and H2 in [14] are easily shown to be correct. Thus we consider the case $\hat{N}_{*} \neq \hat{N}^{*}$.

Theorem 20 When $\hat{N}_{*} \neq \hat{N}^{*}, v$ is not a floating leaf.

Proof. We first assume that $\hat{N}=\hat{N}^{*} \neq \hat{N}_{*}$. Let $S_{1}$ be the section having the leaf $[v]$. If the algorithm uses the template P3 or Q3, the same technique in the proof of Theorem 4.3 in [14] works: Since the label of $\hat{N}$ is 0 or $1, B \neq \emptyset$ in the templates, and $S_{1}$ is not empty by Observation 17 . Thus there are no other place that $[v]$ can be put into. Thus we assume that the algorithm uses the template Q5. Since $S_{1}$ is not empty by Observation 17, $[v]$ cannot be under the sections in $\hat{N}$ except $S_{i}^{\prime}$. On the other hand, since $B \neq \emptyset,[v]$ cannot be under the sections $A \cup B \cup C \cup S_{1}^{\prime}, A \cup B \cup C \cup S_{2}^{\prime}, \cdots, A \cup B \cup C \cup S_{k}^{\prime}$. Thus, the only possible sections are between $S_{i-1}^{\prime}$ and $S_{i}^{\prime}$ or between $S_{i}^{\prime}$ and $S_{i+1}^{\prime}$. However, by Lemma 18, one of them is prohibited. Thus the place of $[v]$ is uniquely determined, and $v$ is not a floating leaf.

We have the following corollary which corresponds to Corollary 4.4 in [14]:

Corollary 21 When $\hat{N}_{*} \neq \hat{N}^{*}$, all nodes properly between $\hat{N}_{*}$ and $\hat{N}^{*}$ on the path P will become inner sections of a $\mathcal{Q}$-node after embedding of $v$. 


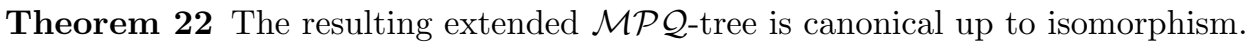

Proof. To derive contradictions, for a given interval probe graph $G$, assume that we have two nonisomorphic trees $T_{1}$ and $T_{2}$ for $G$. We moreover suppose that $G$ has the minimum number of vertices among such graphs. Then there are two vertices $v_{1}$ in $T_{1}$ and $v_{2}$ in $T_{2}$ such that both of $v_{1}$ and $v_{2}$ correspond to $v$ in $G$, and $v_{1}$ and $v_{2}$ guarantee that $T_{1}$ is not isomorphic to $T_{2}$. When $v$ is in $G^{*}$, we immediately have a contradiction to Corollary 7. Thus, $v$ is a simplicial nonprobe in $N_{S}$. If the positions of $v_{1}$ and $v_{2}$ are uniquely determined when they are embedded, we can show that $v_{1}$ and $v_{2}$ have to be embedded in the same place using the same argument in [15], which derives a contradiction. Thus at least one of $v_{1}$ and $v_{2}$ is a floating leaf and embedded in step A3.3. If both of $v_{1}$ and $v_{2}$ are floating leaves embedded in step A3.3, by Observation 15, the positions of $v_{1}$ and $v_{2}$ are the same place since $N\left(v_{1}\right)=N\left(v_{2}\right)$. Thus, without loss of generality, we assume that $v_{1}$ is a floating leaf embedded in A3.3, and $v_{2}$ is embedded in A3.1 or A3.2. However, by Theorem 20, $v_{2}$ is not a floating leaf, which is a contradiction.

\section{Implementation and Complexity}

Theorem 23 For a given interval probe graph $G=(P, N, E)$, let $\tilde{T}$ be the canonical extended $\mathcal{M P} \mathcal{Q}$-tree, and $G^{+}=\left(P, N, E \cup E^{+}\right)$be the corresponding enhanced interval graph. Let $\tilde{E}$ be the set of edges $\left\{v_{1}, v_{2}\right\}$ joining nonprobes $v_{1}$ and $v_{2}$ which is given by $\tilde{T}$; more precisely, we regard $\tilde{T}$ as an ordinary $\mathcal{M P \mathcal { Q }}$-tree, and the graph $\tilde{G}=\left(P \cup N, E \cup E^{+} \cup \tilde{E}\right)$ is the interval graph given by the $\mathcal{M P} \mathcal{Q}$-tree $\tilde{T}$ (thus a floating leaf is not a leaf; the vertex appears in consecutive sections in the corresponding $\mathcal{Q}$-node). Then $\tilde{T}$ can be computed in $O\left((|P|+|N|)|E|+\left|E^{+}\right|+|\tilde{E}|\right)$ time and $O\left(|P|+|N|+|E|+\left|E^{+}\right|+|\tilde{E}|\right)$ space.

Proof. Let $\Delta$ be the maximum degree of probes in $G=(P, N, E)$. Then the step A0 can be performed in $O(|P|+|N|+\Delta|E|)$ time and $O\left(|P|+|N|+|E|+\left|E^{+}\right|\right)$space shown in Appendix A. An enhanced interval probe graph is chordal graph [21,23]. Thus, using Lemma 1, all simplicial nonprobes can be found in linear time and space. Thus the step A1 can be performed in linear time and space. The step A2 can be performed in $O\left(|P|+\left|N^{*}\right|+\left|E^{*}\right|\right)$ time and space. The implementation of step A3 is based on the algorithms in [3, 14]. When the vertex $v$ is not a floating leaf, the algorithm deals with the new set $C$ with the set $B$, and the additional process requires $O(1)$ time and space per iteration with modified implementations in [3, 14]. Thus, its total running time and space for such vertices would be $O\left(|P \cup N|+\left|E \cup E^{+} \cup \tilde{E}\right|\right)$ in amortized manner (see [3, 14] for further details). When the vertex $v$ is a floating leaf, the algorithm checks if the one of the conditions (a), (b), (c), (d) in Section 3.2.1 holds for the section $S_{i}$ for each $i$ with $1 \leq i \leq k$. Each check at section $S_{i}$ can be performed in $O(\operatorname{deg}(v))$ time, and $k=O(|P|+|N|)$ in general. Thus this step requires $O((|P|+|N|) \operatorname{deg}(v))$ time for each nonprobe in $N_{S}$. Hence the total running time required to deal with floating leaves through the algorithm is bounded above by $O((|P|+|N|)|E|)$. Therefore we have the theorem.

Corollary 24 The graph isomorphism problem for the class of (enhanced) interval probe graphs $G$ is solvable in $O\left(n^{2}+n m\right)$ time and $O\left(n^{2}\right)$ space, where $n$ and $m$ are the number of vertices and edges of an affirmative interval graph of $G$, respectively.

Proof. By Theorem 22, for given (enhanced) interval probe graphs $G_{1}$ and $G_{2}, G_{1} \sim G_{2}$ if and only if their

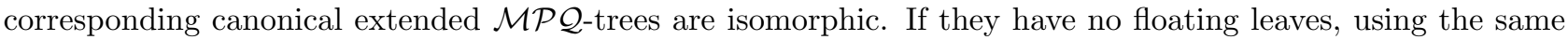
technique in $[15,6]$, the graph isomorphism problem can be solved in linear time. Even if they have floating leaves, it is not difficult to see that comparing two floating leaves $v$ in $G_{1}$ and $G_{2}$ can be done in $O(\operatorname{deg}(v))$ time, which completes the proof.

We note that $\left|E^{+}\right|+|\tilde{E}|$ can be $\Theta\left(|N|^{2}\right)=\Theta\left(n^{2}\right)$ even if $|E|=O(n)$, where $n=|P|+|N|$. Thus the running time in the main theorem can be $\Theta\left(n^{3}\right)$ even if given interval probe graphs have $O(n)$ edges.

\section{Applications}

Given canonical extended $\mathcal{M P Q}$-tree $\tilde{T}$, using a standard depth first search technique, we can compute in linear time if each subtree in $\tilde{T}$ contains only nonprobes. Thus, hereafter, we assume that each section $S_{i}$ knows if its subtree contains only nonprobes or not. 


\subsection{Relations between nonprobes}

We first consider the following problem:

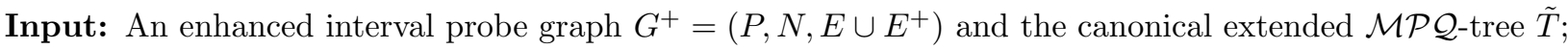

Output: Mapping $f$ from each pair of nonprobes $u, v$ with $\{u, v\} \notin E^{+}$to "intersecting", "potentially intersecting", or "independent";

We denote by $E_{i}$ and $E_{p}$ the sets of the pairs of intersecting nonprobes, and the pairs of potentially intersecting nonprobes, respectively. That is, each pair of nonprobes $u, v$ is either in $E^{+}, E_{i}, E_{p}$, or otherwise, they are independent.

Theorem 25 The sets $E_{i}$ and $E_{p}$ can be computed in $O\left(|E|+\left|E^{+}\right|+\left|E_{i}\right|+\left|E_{p}\right|\right)$ time for a given enhanced interval probe graph $G^{+}=\left(P, N, E \cup E^{+}\right)$and the extended $\mathcal{M P} \mathcal{Q}$-tree $\tilde{T}$.

Proof. We first analyze the relation between two nonprobes. Let $u$ and $v$ be two nonprobes such that $d e p(u) \leq$ $\operatorname{dep}(v), u$ is in node $\hat{N}_{u}$, and $v$ is in node $\hat{N}_{v}$. When $\hat{N}_{u}$ (and $\hat{N}_{v}$ ) is a $\mathcal{Q}$-node, we assume that $u$ appears from $S_{u l}$ to $S_{u r}$ (and $v$ appears from $S_{v l}$ to $S_{v r}$, respectively). There are three cases we have to consider:

(1) $\hat{N}_{u}=\hat{N}_{v}$. If $\hat{N}_{u}\left(=\hat{N}_{v}\right)$ is a leaf, by Assertion $14(2)$, both of $u$ and $v$ are in $N^{*}$. Moreover, by Theorem 2(d), $u$ and $v$ are simplicial. Thus $u$ and $v$ are in $N_{S}$, which is a contradiction. Thus $\hat{N}_{u}$ is not a leaf. If $\hat{N}_{u}$ is a $\mathcal{P}$-node, it has at least two subtrees $T_{1}$ and $T_{2}$ under $\hat{N}_{u}$. If both of $T_{1}$ and $T_{2}$ contain probes, we have $\{u, v\} \in E^{+}$, which contradicts that $\{u, v\} \notin E^{+}$. Thus at most one subtree $T_{1}$ under $\hat{N}_{u}$ contains probes, and all probes in $T_{1}$ intersect with each other. We first assume that such subtree $T_{1}$ containing probes exists.

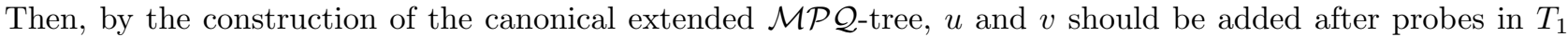
and they should be embedded as leaves under $T_{1}$, which is a contradiction. Thus all subtrees of $\hat{N}_{u}$ contain only nonprobes, which also contradicts Assertion 14(1). Therefore $\hat{N}_{u}$ is not a $\mathcal{P}$-node. If $\hat{N}_{u}$ is a $\mathcal{Q}$-node, we have three subcases.

(1-1) Neither $u$ nor $v$ are floating leaves. In the case, $\{u, v\} \in E_{i}$ if and only if they share common sections; that is, $\max \{u l, v l\} \leq \min \{u r, v r\}$. If $\max \{u l, v l\}-1=\min \{u r, v r\},\{u, v\} \in E_{p}$; otherwise, $u$ and $v$ are independent.

(1-2) $u$ is a floating leaf, and $v$ is not (symmetric case is omitted). Let $\mathcal{S}_{u}$ be the set of sections that $u$ can hang down, and $\mathcal{S}_{v}$ be the set of sections between $S_{v l}$ and $S_{v r}$. If $\mathcal{S}_{u} \subseteq \mathcal{S}_{v}$, we have $\{u, v\} \in E_{i}$. If $\mathcal{S}_{u} \cap \mathcal{S}_{v} \neq \emptyset$ and $\mathcal{S}_{u} \backslash \mathcal{S}_{v} \neq \emptyset$, we have $\{u, v\} \in E_{p}$. Otherwise $\left(\mathcal{S}_{u} \cap \mathcal{S}_{v}=\emptyset\right)$, they are independent.

(1-3) Both of $u$ and $v$ are floating leaves. Let $\mathcal{S}_{u}$ and $\mathcal{S}_{v}$ be the sets of sections that $u$ and $v$ can hang down, respectively. In the case, $\{u, v\} \in E_{p}$ if and only if $\mathcal{S}_{u} \cap \mathcal{S}_{v} \neq \emptyset$. Otherwise, they are independent.

(2) $\hat{N}_{u}$ is an ancestor of $\hat{N}_{v}$ (symmetric case is omitted). If $\hat{N}_{u}$ is a $\mathcal{P}$-node, clearly, $\{u, v\} \in E_{i}$. Thus we assume that $\hat{N}_{u}$ is a $\mathcal{Q}$-node, and $\hat{N}_{v}$ is in the subtree $T_{i}$ under the section $S_{i}$ in $\hat{N}_{u}$.

We first assume that $u$ is not a floating leaf. Then, $\{u, v\} \in E_{i}$ if and only if $u l \leq i \leq u r$. If $i<u l-1$ or $i>u r+1$, they are independent. We assume that $i=u l-1$ or $i=u r+1$. In the case, intuitively, if the vertex $v$ can appear in the leftmost or rightmost node in $T_{i},\{u, v\}$ is in $E_{p}$, or otherwise, they are independent. To check this, we use the following procedure $\operatorname{Adj}\left(\hat{N}_{u}, v\right)$, and we have $\{u, v\} \in E_{p}$ if it returns "Yes", or otherwise they are independent:

Procedure $\operatorname{Adj}\left(\hat{N}_{u}, v\right)$ :

(1) for each node $\hat{N}$ on the path joining the nodes $\hat{N}_{u}, \cdots, \hat{N}_{v}$, do the following;

(1.1) if $\hat{N}=\hat{N}_{u}$, proceed to the next node;

(1.2) if $\hat{N}$ is a $\mathcal{P}$-node and $\hat{N} \neq \hat{N}_{v}$, proceed to the next node;

(1.3) if $\hat{N}$ is a $\mathcal{Q}$-node and $\hat{N} \neq \hat{N}_{v}$, do the following;

(1.3.1) let $S_{i}$ be the section in $\hat{N}$ such that its subtree contains $\hat{N}_{v}$, and let $k$ be the number of sections in $\hat{N}$

(1.3.2) if all sections $S_{1}, S_{2}, \cdots, S_{i-1}$ have subtrees containing only nonprobes, and all probes in sections $S_{1}, S_{2}, \cdots, S_{i-1}$ are contained in $S_{i}$, or its symmetric case holds, proceed to the next node; otherwise, return "No";

(1.4) if $\hat{N}=\hat{N}_{v}$, do the following; 
(1.4.1) if $\hat{N}$ is a leaf or a $\mathcal{P}$-node, return "Yes";

(1.4.2) if $\hat{N}$ is a $\mathcal{Q}$-node with sections $S_{1}, \cdots, S_{k}$, do the following;

(1.4.2.1) let $S_{i}$ be the section in $\hat{N}$ that contains $L(v)$, and $S_{j}$ be the section in $\hat{N}$ that contains $R(v)$;

(1.4.2.2) if $i=1$ or $j=k$ then return "Yes";

(1.4.2.3) if all sections $S_{1}, S_{2}, \cdots, S_{i-1}$ have subtrees containing only nonprobes, and all probes in sections $S_{1}, S_{2}, \cdots, S_{i-1}$ are contained in $S_{i}$, or its symmetric case holds, return "Yes", otherwise, return "No".

We next assume that $u$ is a floating leaf. If $u$ cannot hang down $S_{i-1}, S_{i}, S_{i+1}$, the section between $S_{i-1}$ and $S_{i}$, and the section between $S_{i}$ and $S_{i+1}$, clearly, $u$ and $v$ are independent. Thus we assume that $u$ can hang down at least one of those sections. If the subtree of $S_{i}$ contains only nonprobes, by Assertion 14(1) and (2), $\{u, v\} \in E_{p}$. Thus, we assume that the subtree of $S_{i}$ contains not only nonprobes, but also probes $w$. In the case, $w \notin N(u)$. Thus we have $\{u, v\} \in E_{p}$ if and only if there are arrangements of intervals such that the interval $[\min \{R(u), R(v)\}, \max \{L(u), L(v)\}]$ contains no $R(w) \mathrm{s}$ and $L(w)$ s for each probe $w$ in the subtree. More precisely, $\{u, v\} \in E_{p}$ if and only if $\operatorname{Adj}\left(\hat{N}_{u}, v\right)$ returns "Yes", or otherwise, $u$ and $v$ are independent.

(3) $\hat{N}_{u}$ is not an ancestor of $\hat{N}_{v}$, and $\hat{N}_{v}$ is not an ancestor of $\hat{N}_{u}$. Let $\hat{N}_{c}$ be the nearest common ancestor of $\hat{N}_{v}$ and $\hat{N}_{u}$. In the case, $\{u, v\} \in E_{p}$ if and only if there are arrangements of intervals such that the interval $[\min \{R(u), R(v)\}, \max \{L(u), L(v)\}]$ contains no $R(w) \mathrm{s}$ and $L(w)$ s for each probe $w$ in the subtree rooted at $\hat{N}_{c}$. Thus we can determine if $\{u, v\} \in E_{p}$ using the following algorithm:

(1) If $\hat{N}_{c}$ is a $\mathcal{P}$-node, $\{u, v\} \in E_{p}$ if and only if both of $\operatorname{Adj}\left(\hat{N}_{c}, u\right)$ and $\operatorname{Adj}\left(\hat{N}_{c}, v\right)$ return "Yes", otherwise, $u$ and $v$ are independent.

(2) If $\hat{N}_{c}$ is a $\mathcal{Q}$-node, let $S_{i}$ and $S_{j}$ be the sections in $\hat{N}_{c}$ such that $S_{i}$ and $S_{j}$ contain $\hat{N}_{v}$ and $\hat{N}_{u}$, respectively. Without loss of generality, we assume that $i<j$. Then, $\{u, v\} \in E_{p}$ if and only if both of $\operatorname{Adj}\left(\hat{N}_{c}, u\right)$ and $\operatorname{Adj}\left(\hat{N}_{c}, v\right)$ return "Yes", and either (a) $j-i=1$ or (b) all sections $S_{i+1}, \cdots, S_{j-1}$ have subtrees containing only nonprobes, and $S_{i} \cap P=S_{i+1} \cap P=\cdots=S_{j-1} \cap P=S_{j} \cap P$. Otherwise, $u$ and $v$ are independent.

Now we prove the theorem. The correctness of the above analysis can be done by the induction for the length of the path(s) between $\hat{N}_{u}$ and $\hat{N}_{v}$; which is straightforward but the details are rather tedious, and therefore omitted. Using the standard dynamic programming technique from the leaves to the root, those relations can be computed in $O\left(|E|+\left|E^{+}\right|+\left|E_{i}\right|+\left|E_{p}\right|\right)$ time and space.

By Theorem 25, we can heuristically find the "best" nonprobe to fix the structure of the DNA sequence:

Corollary 26 For a given enhanced interval probe graph $G^{+}=\left(P, N, E \cup E^{+}\right)$and the canonical extended $\mathcal{M P Q}$-tree $\tilde{T}$, we can find the nonprobe $v$ that has most potentially intersecting nonprobes in $O\left(|E|+\left|E^{+}\right|+\right.$ $\left.\left|E_{i}\right|+\left|E_{p}\right|\right)$ time.

\subsection{Enumeration of all affirmative interval representations}

We next consider the following problem:

Input: An interval probe graph $G=(P, N, E)$ and the canonical extended $\mathcal{M P} \mathcal{Q}$-tree $\tilde{T}$;

Output: All affirmative interval graphs.

Theorem 27 For a given enhanced interval probe graph $G=(P, N, E)$ and the canonical extended $\mathcal{M P} \mathcal{Q}$-tree $\tilde{T}$, all affirmative interval graphs can be enumerated in polynomial time and space of $|P|+|N|+|M|$, where $M$ is the number of the affirmative interval graphs.

Proof. We here show how to generate one possible affirmative interval graph of $G$. It is easy to modify it to enumerate all affirmative interval graphs in polynomial time and space of $|P|+|N|+|M|$. We first fix each floating leaf as a leaf under the corresponding $\mathcal{Q}$-node (in arbitrary way). Then, we have an affirmative $\mathcal{M P Q}$-tree for some interval graph. However, to generate all possible interval graphs, we have to consider two more cases; (1) two adjacent nonprobes in $N^{*}$ might have intersection as noted in Observation 8, and (2) two adjacent nonprobes in $N_{S}$ might have intersection as noted in Assertion 14(1). Those two cases can be analyzed 
in the same case-analysis in the proof of Theorem 25. Then we next fix the relations between each pair of nonprobes (We note that some pair of nonprobes $u$ and $v$ may be determined by the relation of the other pair of nonprobes $u$ and $w$ ). It is easy to see that for each possible affirmative interval graph, its $\mathcal{M P} \mathcal{Q}$-tree can be generated in this way.

\section{Concluding Remarks}

It may seem to be straightforward to modify the algorithm to solve the recognition problem for interval probe graphs (with its vertex partition). However, it is not true. Our algorithm does not mind the consistency of the floating leaves. In section 3.2.1, let us suppose there are many floating leaves $v_{i}$ that satisfy the condition (d); $S_{j} \cap S_{j+1} \cap P \subset A_{i} \subset S_{j} \cap P$ for some fixed $j$ and $i=1,2, \cdots$. In the case, we have to check if they can be linearly sorted in inclusion at this point. The check of the consistency can be solved in $O\left(n^{2}\right)$ time. Thus it is possible to modify our algorithm to solve the recognition problem for the (enhanced) interval probe graphs; but the algorithms in $[13,16,12]$ are faster.

\section{References}

[1] C. Berge. Hypergraphs. Elsevier, 1989.

[2] J.R.S. Blair and B. Peyton. An Introduction to Chordal Graphs and Clique Trees. In Graph Theory and Sparse Matrix Computation, volume 56 of IMA, pages 1-29. (Ed. A. George and J.R. Gilbert and J.W.H. Liu), Springer, 1993.

[3] K.S. Booth and G.S. Lueker. Testing for the Consecutive Ones Property, Interval Graphs, and Graph Planarity Using PQ-Tree Algorithms. Journal of Computer and System Sciences, 13:335-379, 1976.

[4] A. Brandstädt, F.F. Dragan, H.-O. Le, V.B. Le, and R. Uehara. Tree Spanners for Bipartite Graphs and Probe Interval Graphs. In 29th International Workshop on Graph-Theoretic Concepts in Computer Science (WG '03), pages 106-118. Lecture Notes in Computer Science Vol. 2880, Springer-Verlag, 2003.

[5] A. Brandstädt, V.B. Le, and J.P. Spinrad. Graph Classes: A Survey. SIAM, 1999.

[6] C.J. Colbourn and K.S. Booth. Linear Time Automorphism Algorithms for Trees, Interval Graphs, and Planar Graphs. SIAM Journal on Computing, 10(1):203-225, 1981.

[7] P. Galinier, M.Habib, and C. Paul. Chordal Graphs and Their Clique Graphs. In WG '95, pages 358-371. Lecture Notes in Computer Science Vol. 1017, Springer-Verlag, 1995.

[8] M.C. Golumbic. Algorithmic Graph Theory and Perfect Graphs. Academic Press, 1980.

[9] M.C. Golumbic and M. Lipshteyn. Chordal Probe Graphs. In WG 2003, pages 249-260. Lecture Notes in Computer Science Vol. 2880, Springer-Verlag, 2003.

[10] M.C. Golumbic and A.N. Trenk. Tolerance Graphs. Cambridge studies in advanced mathematics 89. Cambridge, 2004.

[11] W.-L. Hsu and T.-H. Ma. Substitution Decomposition on Chordal Graphs and Applications. In ISA '91, pages 52-60. Lecture Notes in Computer Science Vol. 557, Springer-Verlag, 1991.

[12] J.L. Johnson, R.M. McConnell, and J.P. Spinrad. Linear Time Recognition of Probe Interval Graphs. in preparation, 2002.

[13] J.L. Johnson and J.P. Spinrad. A Polynomial Time Recognition Algorithm for Probe Interval Graphs. In Proc. 12th Ann. ACM-SIAM Symp. on Discrete Algorithms, pages 477-486. ACM, 2001.

[14] N. Korte and R.H. Möhring. An Incremental Linear-Time Algorithm for Recognizing Interval Graphs. SIAM Journal on Computing, 18(1):68-81, 1989. 
[15] G.S. Lueker and K.S. Booth. A Linear Time Algorithm for Deciding Interval Graph Isomorphism. Journal of the ACM, 26(2):183-195, 1979.

[16] R.M. McConnell and J.P. Spinrad. Construction of Probe Interval Models. In Proc. 13th Ann. ACM-SIAM Symp. on Discrete Algorithms, pages 866-875. ACM, 2002.

[17] T.A. McKee and F.R. McMorris. Topics in Intersection Graph Theory. SIAM, 1999.

[18] F.R. McMorris, C. Wang, and P. Zhang. On Probe Interval Graphs. Discrete Applied Mathematics, 88:315-324, 1998.

[19] T. Nagoya, R. Uehara, and S. Toda. Completeness of Graph Isomorphism Problem for Bipartite Graph Classes. In IEICE Technical Report, volume COMP2001-93, pages 1-5, 3/12 2002.

[20] A. Nerry, M.C. Golumbic, and M. Lipshteyn. Two Tricks to Triangulate Chordal Probe Graphs in Polynomial Time. In Proc. 15th Ann. ACM-SIAM Symp. on Discrete Algorithms, pages 955-962. ACM, 2004.

[21] P. Zhang. Probe Interval Graphs and Its Applications to Physical Mapping of DNA. manuscript, 1994.

[22] P. Zhang. Probe Interval Graph and Its Applications to Physical Mapping of DNA. RECOMB 2000, Poster Session; available at http://recomb2000.ims.u-tokyo.ac.jp/Posters/list-posters.html, 2000.

[23] P. Zhang. United States Patent. Method of Mapping DNA Fragments. [Online] Available http://www.cc.columbia.edu/cu/cie/techlists/patents/5667970.htm, July 32000.

\section{A Computing Enhanced Edges}

In this section, we show an efficient algorithm for generating enhanced edges. More precisely, for a given interval probe graph $G=(P, N, E)$, the set $E^{+}$of enhanced edges can be computed in $O(|P|+|N|+\Delta|E|)$ time and $O\left(|P|+|N|+|E|+\left|E^{+}\right|\right)$space, where $\Delta$ is the maximum degree of probes in $P$. We note that $\left|E^{+}\right|$can be $\Theta\left((|P|+|N|)^{2}\right)$ even if $|E|=O(|P|+|N|)$ in general. The algorithm contains two phases.

First, the algorithm constructs one of possible interval representations $\mathcal{I}=\left\{I_{1}, \cdots, I_{n_{1}}\right\}$ of the interval graph $G[P]$, which runs in $O(|P|+|E|)$ time and space (see, e.g., [14]). Without loss of generality, we assume that the intervals do not share common endpoints, and each endpoint is an integer in $[1,2|P|]$. We note that $G[P]$ is not connected in general, while $G=(P, N, E)$ is connected.

Then the second phase of the algorithm is the following:

C0. set $E^{+}:=\emptyset$;

C1. for each $i=1,2, \cdots, 2|P|$ do the following;

C1.1. if $i=R\left(I_{v}\right)$ for some $I_{v}$, take each pair of nonprobes $\left\{u_{1}, u_{2}\right\}$ with $u_{1}, u_{2} \in N(v)$, and record "the pair $\left\{u_{1}, u_{2}\right\}$ is a candidate";

C1.2. if $i=L\left(I_{v}\right)$ for some $I_{v}$, take each pair of nonprobes $\left\{u_{1}, u_{2}\right\}$ with $u_{1}, u_{2} \in N(v)$, and if the pair $\left\{u_{1}, u_{2}\right\}$ is a candidate, add it into $E^{+}$;

Theorem 28 The algorithm finds all enhanced edges in $O(|P|+|N|+\Delta|E|)$ time and $O\left(|P|+|N|+|E|+\left|E^{+}\right|\right)$ space.

Proof. We first show the correctness. Let $u$ and $u^{\prime}$ be nonprobes with $\left\{u, u^{\prime}\right\} \in E^{+}$. Then there are two independent probes $v$ and $v^{\prime}$ such that $I_{v} \cap I_{u} \neq \emptyset, I_{v^{\prime}} \cap I_{u} \neq \emptyset, I_{v} \cap I_{u^{\prime}} \neq \emptyset$, and $I_{v^{\prime}} \cap I_{u^{\prime}} \neq \emptyset$. Then, in any fixed interval representations of $G, v$ and $v^{\prime}$ are independent. Without loss of generality, we assume that $R(v)<L\left(v^{\prime}\right)$ in the interval representation. Then, when $i=R(v)$ in step C1.1, the algorithm stores $\left\{u, u^{\prime}\right\}$ as a candidate, and when $i=L\left(v^{\prime}\right)$, the algorithm adds $\left\{u, u^{\prime}\right\}$ into $E^{+}$. It is easy to see that the edges added into $E^{+}$are all enhanced edges, which completes the proof of the correctness of the algorithm.

Next we analyze complexity. In the implementation, in step C1.1, each vertex $u$ in $N(v)$ keeps " $u$ is marked by $v$ as candidates". In step C1.2, the algorithm first collects all vertices $u$ in $N(v)$, and check the set of vertices marked by a common vertex $v^{\prime}$ for each $v^{\prime}$ in $N(u)$. It is easy to check the algorithm requires $O(|P|+|N|+$ $\left.|E|+\left|E^{+}\right|\right)$space. For each $i$, step C1.1 runs in $O(\operatorname{deg}(v))$ time, and step C1.2 runs in $O\left(\sum_{u \in N(v)} \operatorname{deg}(u)\right)$. Thus the total running time of the algorithm is $\sum_{v \in P}\left(O(\operatorname{deg}(v))+O\left(\sum_{u \in N(v)} \operatorname{deg}(u)\right)\right)=O(|P|+|N|+\Delta|E|)$ time. 